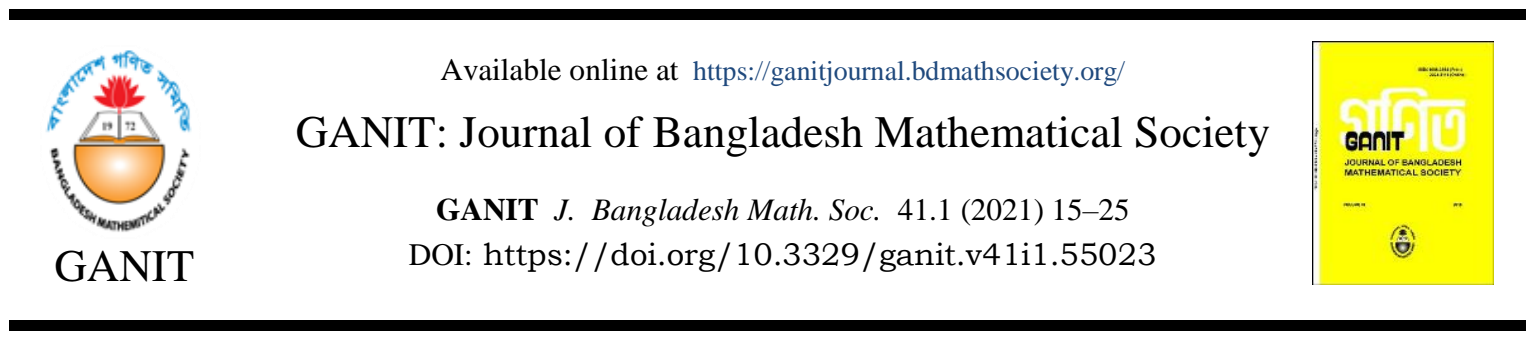

\title{
Factorizing a Quadratic Expression with Reasoning
}

\author{
Anwar H. Joarder * \\ Department of CSE, Northern University of Business and Technology Khulna, \\ Khulna 9100, Bangladesh.
}

\begin{abstract}
It is not popularly realized that the factorization of a general quadratic expression basically requires solution to a hyperbola and a line. This fact is conspicuously pointed out and a few solutions to the problem are demonstrated that are scattered in the literature. In the high school level, the coefficients of a quadratic expression are mostly integers, and factorization is performed by the popular method of decomposition of the middle term. In this expository note, we have presented it in a simpler way that shows both insight and reasoning into the problem. Some other methods, namely, Linear Method, Average Method and Difference of two Squares Method are also discussed. Depending on the background, a reader may prefer a particular method to the others. The expository nature and artistry in presentation of the paper are expected to make the learning of the topic amusing and instructive.
\end{abstract}

(C) 2021 Published by Bangladesh Mathematical Society

Received: November 26, 2020 Accepted: March 18, 2021 Published Online: August 04, 2021

Keywords: Quadratic expression; polynomial of degree 2; factorization.

AMS Subject Classification 2020: 00-01; 00-02; 00A06

\section{Introduction}

Factorization of a quadratic expression or solving a quadratic equation pops up often in physics and engineering for calculating trajectories, and even in sports. If, while watching the Super Bowl, you want to estimate how far a pass thrown by a football quarterback travelled through the air, you solve a quadratic equation. A popular example of a quadratic equation appears at minimizing cost and maximizing profit in business.

The quadratic expression or its solution has a long history stretching as far back as the Old Babylonian Period around 2000-1600 B.C. See, for example, [1] and [2]. Over four millennia, many mathematicians have contributed to the topic. In particular, several books have surveyed the topic of the quadratic formula, such as Chapter 2 of [3], and mathematical history books such as [4], [5] and [1]. For a brief description see [6] and the references therein.

\footnotetext{
*Corresponding author: Anwar H. Joarder, E-mail address: ajstat@gmail.com
} 
The general quadratic expression $q(x)=a x^{2}+b x+c$ with leading coefficient $a \neq 0$ may be termed as a polynomial of degree 2 in the variable $x$ or briefly a quadratic trinomial. The quantities $a, b$ and $c$ are coefficients of the quadratic expression. The quadratic expression with leading coefficient $a=1$, i.e., $x^{2}+b x+c$ is called a monic quadratic expression. Factorization of a quadratic expression appears at algebra at high school level and it is usually done by decomposing the middle term. But broad spectrum of students still feel a significant leap of insight. While factorization of the monic quadratic expression is relatively easier, its connection with the general quadratic expression is not usually spelled out to the students during factorization. See for example [7].

It is easy to check that $(y+j)(y+k)=y^{2}+(j+k) y+j k$ which is a monic quadratic expression as the coefficient of $y^{2}$ is 1 . We can write a standard monic quadratic expression as $y^{2}+b y+m$. Then its factorization is a historic problem of determining $j$ and $k$ given the sum $j+k=b$ and the product $j k=m$. While there are many solutions to the problem, the most popular is the decomposition of the middle term, i.e. given $b$, we try to find $j$ and $k$ such that $j k=m$. In case $j$ and $k$ are numbers, then $j+k=b$ and $j k=m$ are a line and a hyperbola whose precise graphical solution requires a computer. At the middle school level, $j$ and $k$ are usually integers, then $j+k=b$ and $j k=m$ are a dotted line and a dotted hyperbola.

It is not popularly realized that the factorization of a general quadratic expression basically requires solution to a line and a hyperbola. This fact is pointed out clearly for students of secondary or tertiary education and written at that level of simplicity. It is amazing that in some cases when the coefficients are integers, the dotted line and dotted hyperbola have a simple solution.

The paper is organized as follows. Section 2 shows the connection between a general quadratic expression and a monic quadratic expression while factorization. The solution to find two numbers given their sum and product, was known to the Babylonians. See, for example, [1], [3], [4], [8] and [18]. The method of Additive Decomposition of the Middle Term $b$ is explained in Section 3 in an instructive way when the coefficients are integers. It is renamed as Factoring Method.

Two other methods namely, Linear Method and Average Method are also discussed in Sections 4 and 5 respectively. The average method is due to Babylonians but recently popularized by [6,9] providing a clear insight with much simplicity. The method of completing the square by al-Khawarizmi is discussed in Section 6 by characterizing it by the derivative of a quadratic expression and discriminant of the quadratic equation. Because of its importance, many authors have published on the issue with alternative representations. See for example, $[10,11,12,13,14,15,16,17]$.

\section{General and Monic Quadratic Expressions}

The connection between the general quadratic expression and monic quadratic expression required during factorization is spelled out well in this section.

Theorem 2.1 Let

$q(x)=a x^{2}+b x+c, a \neq 0, c \neq 0$,

$(2.1)$

be the quadratic expression. Also let the monic quadratic from $y^{2}+b y+m$ be factorized as

$y^{2}+b y+m=(y+j)(y+k)=Q(y ; j, k)$,

so that $j+k=b$

and $j k=m$. 
Then $q(x)$ can be factorized by the following equivalent ways:

a. $q(x)=\frac{1}{a}(a x+j)(a x+k)$,

b. $q(x)=\frac{1}{a} Q(a x ; j, k)=a Q\left(x ; \frac{j}{a}, \frac{k}{a}\right)=Q\left(\sqrt{a} x ; \frac{j}{\sqrt{a}}, \frac{k}{\sqrt{a}}\right)$,

if $j k=a c$.

Proof. It is easy to check that $a \times q(x)=a^{2} x^{2}+a b x+a c$, can be transformed to $y^{2}+b y+a c$ where $y=a x$. By using (2.2), (2.3) and (2.4), we have 2.5(a). The part 2.5(b) follows from (2.5a).

It seems part 2.5(a) is the simplest. Note that $j$ and $k$ can be found by factorizing $y^{2}+b y+a c$ by (2.2) where $a, b$ and $c$ are available from the given quadratic expression $q(x)$. Then plugging them in (2.5a), we can get the factorized form of $q(x)$. Without any loss of generality, we assume that $a>0$. In case $a<0$, we may factorize $-q=-a x^{2}-b x-c$ so that $-a$, the coefficient of $x^{2}$, is non-negative.

The solution to find numbers given their sum and product, was known to the Babylonians. See for example, $[1,3,4,8,18]$.

\section{Factoring Method ( $j$ and $k$ are integers)}

In Theorem 2.1, we have noticed that for factorization of a monic quadratic expression, $y^{2}+b y+m$, we need $(j, k)$ such that $j+k=b$ and $j k=m$. Usually students stumble to find possible solution to $(j, k)$ such that $j+k=b$, and then look for $(j, k)$ where $j k=m$ by trial and error, and the method is well known as Decomposition of the Middle Term. Since the set $\{(j, k): j+k=b\}$ involves many solutions than that in $\{(j, k): j k=m\}$, we recommend to decompose $j k=m$ first and look for $(j, k)$ such that the dotted line $j+k=b$ is satisfied. In this section, we demonstrate a method that factorizes monic quadratic expression through a tabular representation of factoring $|m|=j k \mid$ first and then $m=j k$. Since $j+k=b$, the larger one of $(j, k)$ in absolute value has the same sign as that of $b$. The method is renamed as Factoring Method for simplicity.

Case 1: Let $(m, b)$ be in the first quadrant of $\mathrm{m}-\mathrm{b}$ axes of co-ordinates, i.e., $m>0$ and $b>0$. Obviously, $m=j k>0$ implies that $j$ and $k$ are of the same sign. Then by virtue of $b=j+k>0$, we have $j>0$ and $k>0$ (first quadrant of $\mathrm{j}-\mathrm{k}$ axes of co-ordinates)

Case 2: Let $(m, b)$ be in the second quadrant of $\mathrm{m}-\mathrm{b}$ axes of co-ordinates, i.e., $m<0$ and $b>0$. Obviously, $m=j k<0$ implies that $j$ and $k$ are of the opposite signs. Then by virtue of $b=j+k>0$, 
one possibility is to have $-k<j<0$, i.e., $j<0$ and $k>0$ ( second quadrant of $\mathrm{j}$-k axes of co-ordinates). Alternatively, by virtue of $b=j+k>0$, we have $-j<k<0$, i.e. $j>0$ and $k<0$ ( fourth quadrant of $\mathrm{j}-\mathrm{k}$ axes of co-ordinates).

Case 3: Let $(m, b)$ be in the third quadrant of $\mathrm{m}-\mathrm{b}$ axes of co-ordinates, i.e., $m<0$ and $b<0$. Obviously, $m=j k<0$ implies that $j$ and $k$ are of the opposite signs. Then by virtue of $b=j+k<0$, one possibility is to have $0<j<-k$, i.e., $j>0$ and $k<0$ (fourth quadrant $\mathrm{j}$-k axes of co-ordinates). Alternatively by virtue of $b=j+k<0$, we have $0<k<-j$, i.e., $j<0$ and $k>0$ (second quadrant $\mathrm{j}$-k axes of coordinates).

Case 4: Let $(m, b)$ be in the fourth quadrant of $\mathrm{m}-\mathrm{b}$ axes of co-ordinates, i.e., $m>0$ and $b<0$. Obviously, $m=j k>0$ implies that $j$ and $k$ are of the same sign. Then by virtue of $b=j+k<0$, and noting that the sign of $b$ is the same as that of $j$ and $k$, we have $j<0$ and $k<0$ (third quadrant of $\mathrm{j}-\mathrm{k}$ axes of coordinates).

The above rules may be summarized to two simple rules:

Rule I: If $m>0$, then both the signs of $(j, k)$ are the same as that of $b$.

Rule II: If $m<0$, then the signs of $(j, k)$ are different; the larger one of $(j, k)$ in absolute value has the same sign as that of $b=j+k$.

Note that $Q 1, Q 2, Q 3$ and $Q 4$ in Table 3.1 denote axes of coordinates of $\mathrm{j}-\mathrm{k}$. Figure 3.1a contains the same information presented in a different style.

Table 3.1: Sign Table for $(j, k)$

\begin{tabular}{|c|l|l|}
\hline$(m, b)$ & $(j, k)$ & Comment on Signs of $(j, k)$ \\
\hline$(+,+)$ & $Q 1:(+,+)$ & The signs are the same as that of $b$ \\
\hline$(-,+)$ & $\begin{array}{l}Q 2:(-,+),-k<j<0 \\
/ Q 4:(+,-),-j<k<0 *\end{array}$ & $\begin{array}{l}\text { The signs are different. The larger one in } \\
\text { absolute value has the same sign as that of } b\end{array}$ \\
\hline$(-,-)$ & $\begin{array}{l}Q 4:(+,-), 0<j<-k \\
/ \mathrm{Q} 2:(-,+), 0<k<-j^{*}\end{array}$ & \\
\hline$(+,-)$ & $Q 3:(-,-)$ & The signs are the same as that of $b$ \\
\hline
\end{tabular}

*We have not considered the asterisk cases in the Factoring Method. 


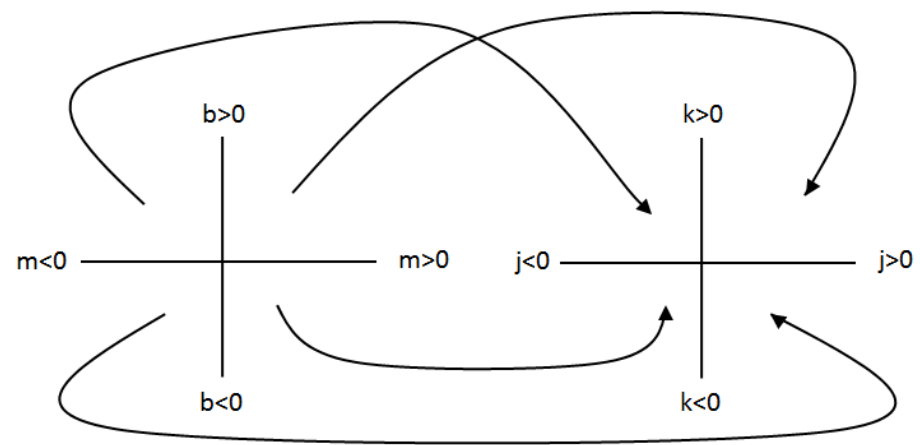

Figure 3.1a Sign table for $(\mathrm{j}, \mathrm{k})$

Keep dividing $|m|=j k \mid$ by possible divisors, say, $j=1,2,3, \ldots$, and then repeating it for $m=j k$ so that it is completely divisible. Stop at $|j| \leq|k|$. Then the solution $(j, k)$ is obvious from their absolute values intuitively or by the above rules. An example is provided below to illustrate the idea.

Example 3.1 Factorize $q(x)=8 x^{2}-2 x-15$.

Solution: It is easy to check that $q(x)=\frac{1}{8}\left(8 x^{2} \times 8-2 x \times 8-15 \times 8\right)$ can be transformed to $\frac{1}{8}\left(y^{2}-2 y-15 \times 8\right)$ where $y=8 x$. We will factorize $y^{2}-2 y-15 \times 8$ first. By comparing it with $y^{2}+b y+m$, we have $m=j k=a c=8(-15)=-120$ and $b=-2$. Obviously, $m=j k<0$ implies that $j$ and $k$ are of the opposite signs. Then by virtue of $b=j+k<0$, one possibility is to have $0<j<-k$, i.e., $j>0$ and $k<0$. Another possibility not used here is $0<k<-j$, i.e., $j<0$ and $k>0$. You may check Case 3 and Rule II at the beginning of this section for general logic or Table 3.1 or Figure 3.1a.

Factoring $|j k|=120$, and then $j k=-120$ by the above arguments or by eye inspection, we have the following table:

Table 3.2: Factoring Table

\begin{tabular}{|l|l|l|l|l|l|l|l|l|}
\hline$j$ & 1 & 2 & 3 & 4 & 5 & 6 & 8 & 10 \\
\hline$k$ & -120 & -60 & -40 & -30 & -24 & -20 & -15 & -12 \\
\hline
\end{tabular}

To satisfy $j+k=-2$, we have $j=10, k=-12$ so that $y^{2}-2 y-15 \times 8=(y+10)(y-12)$ and finally by $(2.5 \mathrm{a})$, we have

$q(x)=\frac{1}{8}(8 x+10)(8 x-12)=(4 x+5)(2 x-3)$.

\section{Linear Method}

In Section 2, we have mentioned that the process of factorization requires to solve a line $j+k=b$ and a hyperbola $j k=m$. The Babylonian tablets clearly indicate that they had realized $j+k=2 b$ and $j k=m$ as the perimeter and the area of a rectangle. These two equations are equivalent to two lines: $k+j=b$ and $k-j=t$ where $t$ is defined in equation (4.1). Thus the problem of factorization boils down to equilibrium 
point of two lines. Algebraically, it means, determining two numbers given their sum and difference. The method may be named as Linear Equilibrium Method or simply Linear Method.

The monic quadratic expression is given by $y^{2}+b y+m$, (say). From Section 2, we have $k+j=b$ and $j k=m$. Assuming $j \leq k$, by virtue of the identity $(k-j)^{2}=(k+j)^{2}-4 j k$, another line $k-j=t$ can be determined where

$t^{2}=b^{2}-4 m$

The solution to the lines $k+j=b$ and $k-j=t$ is

$k=(b+t) / 2$ and $j=(b-t) / 2$.

To factorize $q(x)=8 x^{2}-2 x-15$ of Example 3.1, we have $k+j=-2$ and $j k=-120<0$. Assuming $0<k<-j$, i.e., $k>0$ and $j<0$, or, $k>0$ and $-j>0$, it follows that $k+(-j)>0$. Then $(k-j)^{2}=(k+j)^{2}-4 j k=484$ so that $k-j=22$. Solving $k+j=-2(=b)$ and $k-j=22(=t)$, by equation (4.2), we have $k=[(-2)+22] / 2=10$ and $j=[(-2)-(22)] / 2=-12$. Alternatively by assuming $0<j<-k$, i.e., $j>0$ and $k<0$, we have $k=-12$ and $j=10$. Then the factorization is obvious by (2.5a).

\section{Average Method}

As mentioned earlier, Babylonians were interested in finding the dimensions $j$ and $k$ of a rectangle with a given area $m$ and a given perimeter $2 b$. They solved it by a method what may be called the Average Method and is recently popularized by [6,9]. In general, let the average of the two numbers $j$ and $k$ be $b / 2$. Assume that the smaller number $(j)$ is $r$ units below the average and the larger one $(k \geq j)$ is $r$ units above the average. That is, they are of the form: $j=(b / 2)-r$ and $j=(b / 2)+r$, where $r(\geq 0)$ is yet to be determined. Then by equation (2.4) and (2.6), we have $j k=m=a c$, and hence the following average equation:

$\left(\frac{b}{2}-r\right)\left(\frac{b}{2}+r\right)=a c$,

(5.1) or, $r^{2}=(b / 2)^{2}-a c$,

or, $r=\sqrt{(b / 2)^{2}-a c}$,

where $b^{2} \geq 4 a c$

The beauty of this method is that $r$ has a natural meaning. Moreover since $a, b$ and $c$ are known, $r$ is easily determined. Also in Section 6, this method shows a new meaning of discriminant of a quadratic equation. Algebraically the Linear Method and the Average Method are equivalent but the clarity of natural meaning of $r$ in the Average Method compared to $t$ (see equation 4.2) in the Linear Method deserves appreciation.

To factorize $q(x)=8 x^{2}-2 x-15$ of Example 3.1, we see the average of $j$ and $k$ is $-2 / 2=-1$, and 
they are of the form $j=-1-r$ and $k=-1+r$ so that by the average equation in (5.1), we have $(-1-r)(-1+r)=a c=-120$, or, $r=11$. Hence they are $j=-1-r=-12$ and $k=-1+r=10$. Then the factorization is obvious by equation (2.5a).

Note that it follows from (5.2) that

$2 r=\sqrt{b^{2}-4 a c}=k-j$

Hence one may save time during factorization by utilizing the equation $2 r=\sqrt{b^{2}-4 a c}$. In case $j+k=b$

is not an even number, it would be easier to assume average is $b / 2=B$ so that the average equation (5.1) would be $(B-r)(B+r)=a c$.

\section{Difference of Squares (DS) Method}

In the year 825, about 2500 years after the Babylonian tablets were created, a general method similar to today's Quadratic Formula was authored by the Arab mathematician Muhammad ibn Musa al-Khwarizmi in a book titled Hisab al-jabr w'al-muqabala. His technique was completing the square and more general than those of the Babylonians. The word "al-jabr" in the title of his book led to our modern word "algebra". The word "algorithm" comes from al-Khwarizmi's name.The authors in [19,20,21] explore strategies for teaching the method of completing the square using a geometrical or conceptual approach. The method is characterized here in terms of the derivative of a quadratic form and the discriminant of a quadratic equation.

Theorem 6.1 Let $q(x)=a x^{2}+b x+c, a \neq 0$ be a quadratic expression, $q^{\prime}(x)=2 a x+b$ be its derivative and $d=b^{2}-4 a c$ be the discriminant of the equation $q(x)=0$. Then we have $q(x)=\frac{1}{4 a}\left(q^{\prime}(x)+\sqrt{d}\right)\left(q^{\prime}(x)-\sqrt{d}\right)$.

Proof. Multiplying $q(x)=a x^{2}+b x+c$ by $4 a$, we have $4 a q(x)=4 a^{2} x^{2}+4 a b x+4 a c$. It can be expressed as $4 a q(x)=(2 a x+b)^{2}-\left(b^{2}-4 a c\right)=\left(q^{\prime}(x)\right)^{2}-(\sqrt{d})^{2}$ which simplifies to (6.1).

The above is equivalent to the popular factorization by DS (Difference of Squares). Note that by (5.4), we have

$\sqrt{d}=2 r$.

It exhibits an amazing connection between $r$ and the discriminant $d$ which is implicit in [6].

Example 6.1 Factorize $q(x)=7 x^{2}-6 x-13$.

Solution: Since $a=7, b=-6, c=-13, d=b^{2}-4 a c=400$, by using (6.1), we have $q(x)=\frac{1}{4 \times 7}\left[(2 \times 7 x-6)^{2}-20^{2}\right]=(x+1)(7 x-13)$.

Corollary 6.1 Let $q(x)=a x^{2}+b x+c, a \neq 0$ be a quadratic expression, $s=2 a x+b$ and $2 r$ be defined in the average equation in (5.1) or (5.4). Then we have the following: 
$q(x)=\frac{1}{4 a}(s+2 r)(s-2 r)$.

The equation (6.3) can also be written as $q(x)=\frac{1}{a}((s / 2)+r)((s / 2)-r)$.

\section{An Algorithm for Factorization}

An algorithm for factorization of a quadratic expression is discussed below with Example 6.1 in mind. It is suitable for any student not having exposed to derivative of a quadratic expression or discriminant of the quadratic equation.

STEP 1: Determining $s$

Determine the leading coefficient $a$, the linear coefficient $b$, the constant term $c$ of the quadratic function $q(x)=a x^{2}+b x+c, a \neq 0$ and prepare the following table:

Table 6.1: Determination of $s$

\begin{tabular}{|l|l|l|}
\hline$a=7$ & $b=-6$ & $c=-13$ \\
\hline $2 x$ & 1 & 0 \\
\hline
\end{tabular}

Then multiplying column wise and adding, we have $s=2 a x+b=14 x-6$. Note that the second line of the table is always the same for any quadratic expression $q(x)$.

STEP 2: Determining $r$

Since $b=-6$ and $a c=7(-13)=-91$, from the average equation $((b / 2)-r)((b / 2)+r)=a c$ in (5.1), we have $(-3-r)(-3+r)=-91$, or, $r=10$.

STEP 3: Factorized Form

From (6.3), we have

$$
\begin{aligned}
& q(x)=\frac{1}{4 a}(s+2 r)(s-2 r)=\frac{1}{4 \times 7}(14 x-6+20)(14 x-6-20), \\
& q(x)=(x+1)(7 x-13) .
\end{aligned}
$$

Corollary 6.2 Let $q(x)=a x^{2}+b x+c, a \neq 0$ be a quadratic expression, $s=2 a x+b$ and $2 r$ be the defined in the average equation in (5.1) or (5.4). Then the solution to $q(x)=0$ can be determined by $s=\mp 2 r$.

To solve $7 x^{2}-6 x-13=0$ (see Example 6.1), we have $s=14 x-6$ and $2 r=20$, so that $14 x-6=\mp 20$ and finally we have $x=-1$, or, $x=13 / 7$. 


\section{General Quadratic Equation}

We mentioned in the introduction that the literature is extremely rich in solving a quadratic equation. Here we refer to [22] who has discussed a less known result of the roots of quadratic equation with amazing geometric meaning. See also two recent papers $[23,24]$. The following theorem provides a solution to the general quadratic equation in terms of the coefficients $a, b$ and $r$.

Theorem 7.1 Let $a x^{2}+b x+c=0, a \neq 0$ be the quadratic equation. Then the roots of the equation are $x_{1}=\frac{1}{a}(-b / 2-r)$, or, $x_{2}=\frac{1}{a}(-b / 2+r)$,

where $r$ can be obtained by the average equation in (5.1) or (5.4).

Proof. The solution to the general quadratic equation $a x^{2}+b x+c=0$, with $a \neq 0$ follows from equation (2.5a) as $x_{1}=-j / a$, or, $x_{2}=-k / a$ so that the sum and the product of the roots are $x_{1}+x_{2}=-(j+k) / a$ and $x_{1} x_{2}=j k / a^{2}$ respectively. By (2.3) and (2.6), we have $j+k=b$ and $j k=a c$ so that $x_{1}+x_{2}=-b / a$ and $x_{1} x_{2}=c / a$. Assuming $x_{1} \leq x_{2}$, by virtue of the average equation in (5.1) or (5.4), and the identity $\left(x_{2}-x_{1}\right)^{2}=\left(x_{2}+x_{1}\right)^{2}-4 x_{2} x_{1}$, or, $\left(x_{2}-x_{1}\right)^{2}=\left(b^{2}-4 a c\right) / a^{2}$, we have $x_{2}-x_{1}=2 r / a$. Then by (4.2), it follows that the solution to the general quadratic equation is given by (7.1).

Note that $b / 2$ and $2 r$ are the average and distance of the roots of $x^{2}+b x+a c=0$.

To solve $7 x^{2}-6 x-13=0$ (see Example 6.1), we have $a=7, b=-6, c=-13$ so that $2 r=\sqrt{b^{2}-4 a c}=20$ and hence by equation (7.1), the roots are $x=-1$, or, $x=13 / 7$.

\section{Conclusion}

We have discussed, identified and named a few methods of factorization of a quadratic trinomial that have been developed over four millennia for making learning and teaching more effective. The rectangular method of the Babylonians is equivalent to solving the equilibrium point of a hyperbola and a line as mentioned in Section 1, and Average Method in Section 5. We recommend using the Factoring Method discussed in Section 3 for high school students as it tries to inculcate the reasoning of the problem. Readers motivated by algebra may like the Linear Method in Section 4 and the Average Method in Section 5. The method by

al-Khwarizmi known as Difference of Squares is characterized in Section 6 by the derivative of a quadratic expression and the discriminant of the quadratic equation. Readers motivated by calculus may appreciate it though it has a mnemonic value for every reader. The choice of the method would certainly depend on the background of the reader.

Acknowledgement The author is grateful to the Editor, and also to Professor S.M. Shahidul Islam, Department of Mathematics, Hajee Mohammad Danesh Science and Technology University, Bangladesh for

comments on earlier versions of the manuscript.

\section{References}

[1] Katz, V.J. A History of Mathematics, 3rd ed. Pearson, MA, Boston, USA (2008). 
[2] Robson, E. Mathematics in Ancient Iraq: A Social History. Princeton University Press, Princeton, NJ, USA (2008).

[3] Irving, R.S. Beyond the Quadratic Formula. The Mathematical Association of America, Washington, DC, USA (2013).

[4] Burton, D.M. History of Mathematics: An Introduction, 7th ed. New York, NY: McGraw-Hill, New York, USA (2010).

[5] Derbyshire, J. Unknown Quantity: A Real and Imaginary History of Algebra. Joseph Henry Press, Washington, DC, USA (2006).

[6] Loh, P.S. A simple proof of the quadratic formula. arXiv:1910.06709v2 (2019).

[7] Steckroth, J. A transformational approach to slip-slide factoring. Mathematics Teacher, 109(3):228-234 (2015).

[8] Vi`ete, F. Opera Mathematica. Edited by Frans van Schooten. Reprinted Bonaventure and Abraham Elzevier, Leiden, The Netherlands.1646 (1579).

[9] Savage, J. Factoring quadratics. The Mathematics Teacher, 82:35-36 (1989).

[10] Autrey, M.A. and Austin, J.D. A novel way to factor quadratic polynomials. The Mathematics Teacher, 72(2):127-128 (1979).

[11] Baker, B. Developing a meaningful algorithm for factoring quadratic trinomials. The Mathematics Teacher, 62(8):629-631 (1969).

[12] Cabahug, J.A. The use of Bruner's Modes of representations in teaching factoring second-degree polynomials. International Journal of Education, 1(1):85-103 (2012).

[13] Dogbey, J., Gyening, J. and Kersaint, G. Factorising quadratic trinomials: An alternative approach. Learning and Teaching Mathematics, 2011(10):44-45 (2011).

[14] Erisman, R. J. Factoring trinomials. The Mathematics Teacher, 79:124-129 (1986).

[15] Hirsch, C.R. Finding factors physically. The Mathematics Teacher, 75(5):388-419 (1982).

[16] Kotsopoulos, D. Unravelling student challenges with quadratics: A cognitive approach. Australian Mathematics Teacher, 63(2):19-24 (2007).

[17] Moskol, A. An alternate method for factoring quadratic trinomials. The Mathematics Teacher, 72(9):676-

677 (1979).

[18] Gandz, S. The Origin and Development of the Quadratic Equations in Babylonian, Greek, and Early Arabic Algebra. Osiris, 3:405-557 (1937).

[19] Joarder, A.H. Factorising with geometric insights. Australian Senior Mathematics Journal, 29(1) :25-31 (2015).

[20] Maharaj, A. A geometrical introduction to the method of completing the square. Learning and Teaching Mathematics, 2005 (2):7-9 (2014). 
[21] Samson, D. Completing the square: a conceptual approach. Learning and Teaching Mathematics, 2013 (14):8-11 (2013).

[22] Samson, D. A short quadratic diversion. Learning and Teaching Mathematics, 2018 (24):21-23 (2018).

[23] Makobe, M.L. Alternative quadratic formula. Learning and Teaching Mathematics, 2014 (17): 42 (2014).

[24] Man, Y.K. A simple approach deriving the quadratic formula. Learning and Teaching Mathematics, 2009 (7):34 (2009). 